\title{
Comparison of Standard Technique, Ultrasonography, and Near-Infrared Light in Difficult Peripheral Vascular Access: A Randomized Controlled Trial
}

\author{
Sercan Yalçınlı, MD; ${ }^{1} \odot$ Funda Karbek Akarca; ${ }^{1}$ Özge Can, MD; ${ }^{1} \odot$ İlhan Uz, MD $;{ }^{1}$ Gülbin Konakçı ${ }^{2}$
}

1. Department of Emergency Medicine, Ege University, Izmir, TURKEY

2. Department of Internal Medicine Nursing Faculty of Health Sciences, Izmir Demokrasi University, Izmir, TURKEY

Correspondence:

Funda Karbek Akarca

Ege University Faculty of Medicine

Emergency Department

35100, Izmir/TURKEY

E-mail: fundakarbek@gmail.com

Conflicts of interest/funding: No conflicts of interest declared. This article received no specific grant from any funding agency.

Keywords: catheterization; peripheral; emergency medicine; emergency nursing; spectroscopy; near-infrared; ultrasonography; interventional

Abbreviations:

DVA: difficult vascular access

NIR: near-infrared light

PIVC: peripheral intravenous catheterization

USG: ultrasonography

Received: July 13, 2021

Revised: September 3, 2021

Accepted: September 3, 2021

doi:10.1017/S1049023X21001217

(C) The Author(s), 2021. Published by Cambridge University Press on behalf of the World Association for Disaster and Emergency Medicine. This is an Open Access article, distributed under the terms of the Creative Commons Attribution licence (https:// creativecommons.org/licenses/by/4.0/), which permits unrestricted re-use, distribution, and reproduction in any medium, provided the original work is properly cited.

\section{Abstract}

Objectives: Successful placement of a peripheral intravenous catheter (PIVC) on the first attempt is an important outcome for difficult vascular access (DVA) patients. This study compared standard technique, ultrasonography (USG), and near-infrared light (NIR) in terms of success in the first attempt in patients with DVA.

Methods: This was a prospective, randomized controlled study. The study was conducted in a tertiary care hospital. Emergency department patients who describe DVA history, have no visible or palpable veins, and were assessed by the nurse to have a difficult PIVC were included to study. The PIVC procedure was performed on patients by standard, USG, or NIR device techniques. For all approaches, the success of the first attempt was the primary aim. Total procedure time, the total number of attempts, and the need for rescue intervention were secondary aims.

Results: This study evaluated 270 patients. The first attempt success rates for USG, standard, and NIR methods were 78.9\%, 62.2\%, and 58.9\%, respectively. The rate of first attempt success was higher in patients who underwent USG (USG versus standard, $\mathrm{P}=$ .014; USG versus NIR, $\mathrm{P}=.004$; standard versus $\mathrm{NIR}, \mathrm{P}=.648)$. The total median (IQR) procedure time for USG, standard, and NIR methods was 107 (69-228), 72 (47134), and 82 (61-163) seconds, respectively. The total procedure time was longer in patients undergoing USG (standard versus USG, $\mathrm{P}<.001$; NIR versus USG, $\mathrm{P}=.035$; standard versus NIR, $\mathrm{P}=.055$ ). The total median (IQR) number of attempts of USG, standard, and NIR methods were 1 (1-1), 1 (1-2), and 1 (1-2), respectively. A difference was found among the groups regarding the total number of attempts (USG versus NIR, $\mathrm{P}=.015$; USG versus standard $\mathrm{P}=.108$; standard versus $\mathrm{NIR}, \mathrm{P}=.307)$. No difference was found among groups in terms of the need for rescue methods.

Conclusion: It was found that USG increases the success of the first attempt compared with the standard method and NIR in patients with DVA.

Yalçınlı S, Karbek Akarca F, Can Ö, Uz İ, Konakçı G. Comparison of standard technique, ultrasonography, and near-infrared light in difficult peripheral vascular access: a randomized controlled trial. Prehosp Disaster Med. 2022;37(1):65-70.

\section{Introduction}

Peripheral intravenous catheterization (PIVC) is a frequently performed procedure in emergency medicine practice. Performing procedures on the first attempt is important to reduce patients' pain and anxiety and increase trust in health care personnel. Patient, disease, and practitioner-related factors influence the success of the procedure.

Difficult vascular access (DVA) is often described as a failure to obtain vascular access after two unsuccessful attempts. ${ }^{1-5}$ Patients with non-visible and non-palpable veins, who describe a DVA history based on previous hospital admissions, and are assessed by the experienced nurse as a challenging procedure are presumed to be difficult PIVC. ${ }^{6,7}$

Health care professionals often use the standard methods with at least two attempts when encountering patients with DVA. In case of failure in these, they can seek help from a more experienced health care professional or try again from different anatomical locations. ${ }^{8}$ Another approach with an increasing frequency of application in recent years is bed-side ultrasonography (USG). It is known that the method, which physicians initially applied, was also successfully applied by nurses. ${ }^{9}$ With the advances in technology, one of the 
methods to find a place in daily practice in patients with DVA is the near-infrared light (NIR) device. However, data on the efficacy of adult patients are very limited in the literature on this subject.

Well-designed randomized controlled trials are needed in adult emergency department patients with DVA, comparing standard techniques with alternative methods, investigating the procedure's success in the first attempt. ${ }^{10}$

This study aimed to compare USG, NIR, and standard technique on the first attempt procedure success for PIVC in adult patients with DVA. The second aim was to investigate whether a difference is found among the methods regarding total procedure time, the total number of attempts, and the need for rescue procedures.

\section{Material and Methods}

Study Design and Setting

This prospective, three-armed, randomized controlled trial was conducted from March 2019-February 2020 in an emergency department of an academic tertiary care hospital. The study was started after the approval of the local ethics committee (Approval number: 18-7/32). Written informed consent was obtained from the nurses and patients before participating in the study. This study followed the Strengthening the Reporting of Observational Studies in Epidemiology (STROBE) guidelines (Supplementary File 1; available online only). ${ }^{11}$

The study included patients describing DVA history ( $>$ two trial histories during vascular access on a previous visit), with no visible or palpable veins on the upper extremity, and who were assessed to have a difficult procedure by the senior nurse (according to classification: easy-moderate-difficult). ${ }^{6}$ Patients who did not provide consent, pregnant, $<18$ years of age, and urgent critical intervention needs were excluded.

The study nurses identified patients who needed PIVC in the emergency department and met the study criteria. After obtaining informed consent from the patients, they were included in the study. The PIVC procedure was performed on patients with the method corresponding to the randomization scheme. To establish a standard approach among the procedures, 18-gauge and 20gauge catheters were used for the arm and forearm regions and other regions, respectively. The procedure time was started at the application of the tourniquet by the study personnel. The time was stopped when at least $5 \mathrm{ml}$ of blood was obtained after venipuncture, or at least $5 \mathrm{ml}$ of saline was administered successfully, and the practitioner terminated the procedure. When two attempts were unsuccessful, the third and subsequent attempts were considered rescue interventions, and these interventions were left to the practitioner's discretion.

\section{Study Outcomes}

For all methods, the first attempt success status was the primary outcome. Total procedure time, the total number of attempts, and the need for rescue intervention were secondary outcomes.

\section{Sample Size}

The first attempt success for PIVC is approximately 50\%. ${ }^{12}$ To increase this success to $70 \%$ with 0.05 Type 1 error and 0.80 statistical power, the number of patients to be recruited was calculated as 88 . The study population was planned as a total of 270 patients, with 90 patients in each group.

\section{Randomization}

The randomization scheme was created by the study coordinator. According to the randomization scheme, case report forms were numbered with an equal number for each group (standard, ultrasound, and NIR) and distributed equally to the study nurses. After the participants were included in the study, they were blinded to which procedure would be applied. The study nurses performed the procedure with the corresponding method by randomly pulling the case report form in a closed and invisible envelope when they encountered patients with study inclusion criteria (Figure 1).

\section{Statistical Analysis}

Statistical analyses were performed using the IBM SPSS Statistics for Windows (Version 25.0; IBM Corp.; Armonk, New York USA). The suitability of continuous variables to normal distribution was examined using analytical methods (KolmogorovSmirnov). Descriptive analysis was performed using the median and interquartile range (IQR) for non-normally distributed variables and means and standard deviations (SD) for normally distributed variables. In the comparison of independent groups, KruskalWallis tests were used for non-normally distributed data. The Mann-Whitney $U$ test was performed to test the significance of pairwise differences for multiple comparisons using Bonferroni correction. For categorical variables, the difference among groups was evaluated with the Chi-square test. A P value less than .05 was considered to show a statistical result.

\section{Training of Study Nurses}

The nurses who had over two years of experience in emergency medicine were informed before the study start. Consent was obtained from senior nurses who wanted to participate in the study. A pre-test of ten questions with theoretical and practical content was applied to measure their knowledge level regarding the use of ultrasound and NIR devices. The principles of determining the venous structures in the upper extremity in the transverse and longitudinal planes, measuring the vessel's depth, recognizing the arterial structures, and performing the procedure on the transverse and longitudinal planes on the dummy were questioned for the ultrasound device. For the NIR device, the principles of device usage and the importance of an appropriate angle were questioned.

A score above $80 \%$ in the pre-test was accepted as a success by the study team. Nurses with a test result $\leq 80 \%$ were considered unsuccessful and underwent four hours of theoretical and four hours of practical training. In theoretical training, emergency medicine physicians experienced in the use of USG explained vascular access with a single operator method in the transverse and longitudinal planes. Also, USG device introduction, principles of use, linear probes and features, horizontal and longitudinal planes concepts, determination principles of venous structures, differentiation of veins from artery and nerve structures, antisepsis techniques, and case report form documentation were explained. SonoSite Edge (FUJIFILM SonoSite, Inc.; Bothell, Washington USA) was used for ultrasonographic procedures.

For the NIR device, the device's introduction, principles of use, the distance between the light and area to be treated, and how the device should be positioned were explained. AccuVein AV 400 (AccuVein Inc.; Cold Spring Harbor, New York USA) was used for procedures using NIR.

In practical training, ultrasonographic recognition of the normal venous structures of the upper limb on live models, target vein diameter, skin depth measurement, tracing of the target vein, gain 

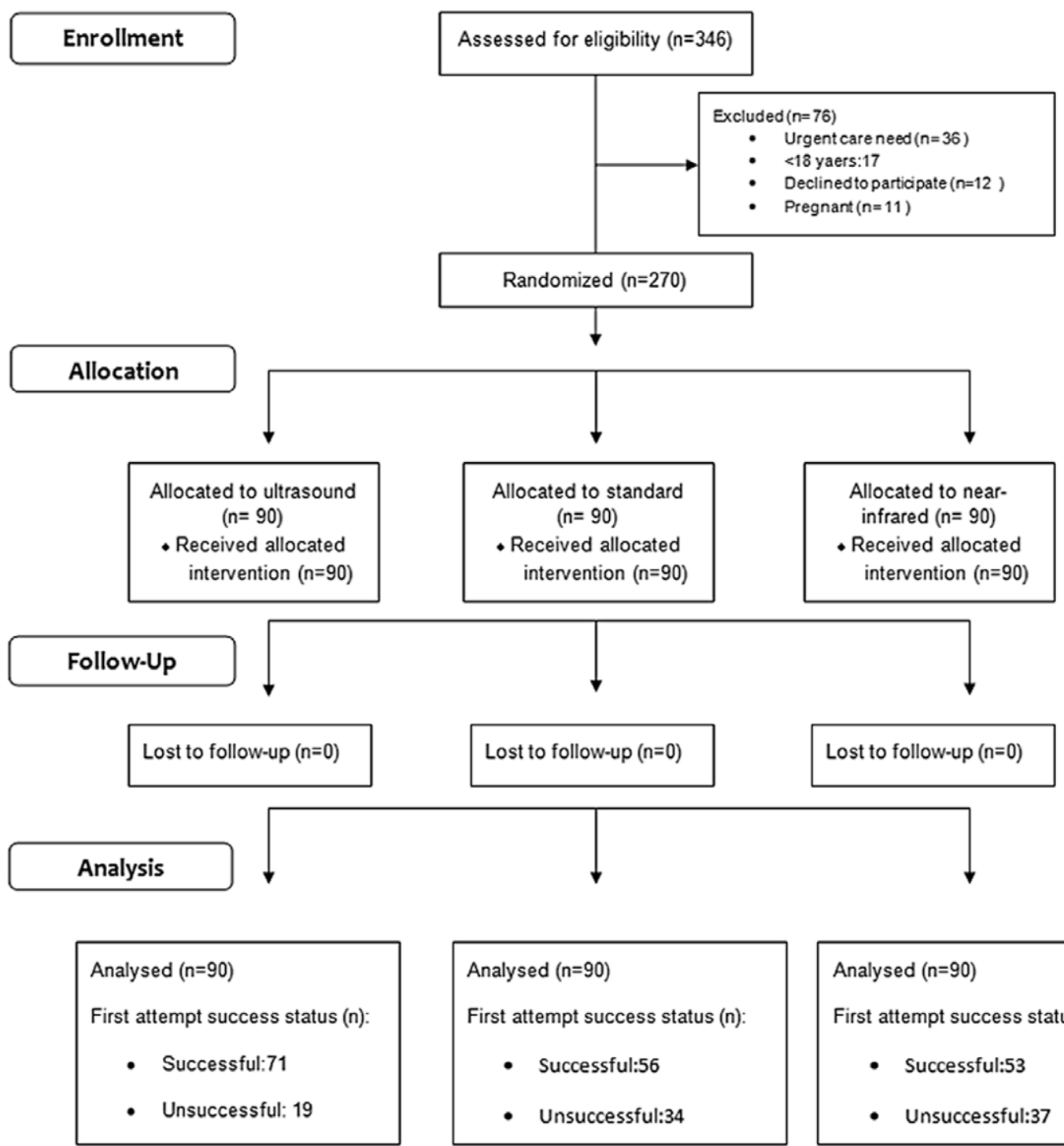

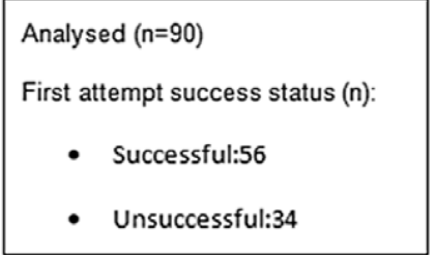

- Unsuccessful:34

Figure 1. Patient Flow Chart and First Attempt Success Status.

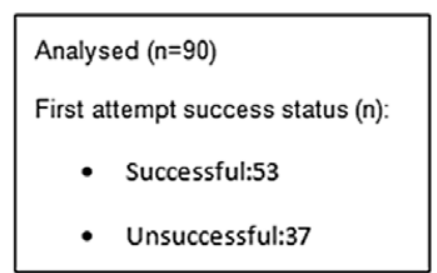

Yalçınlı @ 2022 Prehospital and Disaster Medicine settings, and arterial-vein separation were provided. It was ensured that each nurse performed at least three procedures on horizontal and longitudinal axes ultrasonographically on the forearm mannequin. In the practical training of the NIR device, adjusting the device distance, positioning the device perpendicular to the area to be treated, and identifying the vascular structures while the device was operating were shown.

A post-test that included the same questionnaire in the pre-test was applied to all nurses after theoretical and practical training, and nurses who achieved $\geq 80 \%$ were included in the study. Based on the literature data, each nurse was allowed to obtain vascular access ten times under the supervision of the emergency medicine physician before starting the patient recruitment for research. ${ }^{13}$

\section{Results}

The patient enrollment was conducted from April 2019 through February 2020. Sixteen senior emergency nurses were invited to the study. It was determined that none of the nurses could be successful for the pre-test evaluation. Therefore, all nurses were subjected to theoretical and practical training. The pre-test and post-test scores of these nurses were 29.8 ( $\mathrm{SD}=18.1$; min-max: 10-80) and $76.9(\mathrm{SD}=19.2$; min-max: 41-96). The post-test results of eight nurses were successful. Because two of the eight nurses with sufficient post-test evaluation did not agree to participate, six senior nurses were included in the study. The mean work experience of the nurses included in the study was $7.8(\mathrm{SD}=3.1)$ years (min-max: 5-12).

The study evaluated 270 patients (Figure 1 ). The distribution of age, sex, and DVA criteria were similar among the groups. The most common preferred area for PIVC was the antecubital region $(55.6 \%)$ in all three groups. The demographic information of the patients is shown in Table 1.

The first attempt success was higher in patients who underwent USG (78.9\%) compared with the standard (62.2\%) and infrared (58.9\%) groups. A statistically significant difference was found in success in the first attempt among the groups $(P=.010)$. The total median (IQR) procedure time for USG, standard, and NIR methods was 107 (69-228), 72 (47-134), and 82 (61-163) 


\begin{tabular}{|c|c|c|c|c|c|}
\hline & $\begin{array}{c}\text { Standard } \\
\mathrm{n}: 90\end{array}$ & $\begin{array}{c}\text { Near-Infrared Light } \\
\mathrm{n}: 90\end{array}$ & $\begin{array}{c}\text { Ultrasound } \\
\mathrm{n}: 90\end{array}$ & Total & P Value \\
\hline Age (Median- IQR) & $68.5(51-76)$ & $64(49-77)$ & $64(53-76)$ & $65(51-76)$ & .866 \\
\hline Sex & & & & & .384 \\
\hline Male & $34(37.8)$ & $30(33.3)$ & $39(43.3)$ & $103(38.1)$ & \\
\hline Female & $56(62.2)$ & $60(66.7)$ & $51(56.7)$ & $167(61.9)$ & \\
\hline $\begin{array}{l}\text { Difficult Vascular } \\
\text { Access Criteria of } \\
\text { Patients }\end{array}$ & & & & & .055 \\
\hline $\begin{array}{l}\text { (1) Patient } \\
\text { Statement (>2 } \\
\text { attempts on previous } \\
\text { visit) }\end{array}$ & $9(10.0)$ & $6(6.7)$ & $3(3.3)$ & $18(6.7)$ & \\
\hline (2) Invisible Veins & $2(2.2)$ & $1(1.1)$ & $2(2.2)$ & $5(1.9)$ & \\
\hline $\begin{array}{l}\text { (3) Nurse } \\
\text { Statement (difficult } \\
\text { anticipation) }\end{array}$ & $7(7.8)$ & $2(2.2)$ & $0(0.0)$ & $9(3.3)$ & \\
\hline 1 and 3 & $10(11.1)$ & $6(6.7)$ & $4(4.4)$ & $20(7.4)$ & \\
\hline 1,2 , and 3 & $36(40.0)$ & $46(51.1)$ & $45(50.0)$ & $127(47.0)$ & \\
\hline Total & $90(100.0)$ & $90(100.0)$ & $90(100.0)$ & $270(100.0)$ & \\
\hline
\end{tabular}

Table 1. Demographic Features of Patient Groups

seconds, respectively. Differences were determined among the groups in terms of total procedure time $(\mathrm{P}=.001)$. The procedure time was longer in patients undergoing USG. The total median (IQR) number of attempts of USG, standard, and NIR methods were 1 (1-1), 1 (1-2), and 1 (1-2), respectively. A difference was found in terms of the total number of attempts among the groups $(\mathrm{P}=.046)$. This difference occurred between USG and infrared groups $(\mathrm{P}=.014)$. The total number of attempts did not differ between the USG and standard technique groups $(\mathrm{P}=.141)$. No difference was found among groups in terms of the need for rescue methods $(\mathrm{P}=.250)$. Ultrasonography was the most preferred rescue method among patients (55.9\%). Near-infrared light was the least preferred rescue method among patients (6.8\%). Comparison of study outcomes among patient groups is shown in Table 2.

\section{Discussion}

This is the first study comparing standard technique, NIR, and ultrasound in terms of first attempt success for PIVC in adult patients with DVA. This study found that the first attempt success rate was higher in the ultrasound group than the standard and NIR groups. No difference was found between standard versus USG and standard versus NIR groups regarding the total number of attempts. Still, more attempts were performed in the infrared device group than in the ultrasound group. The duration of the procedure was longer than the other methods in patients using ultrasound. No difference among groups was detected in terms of the need for rescue methods. Ultrasound was preferred more frequently in patients in need of rescue methods.

The first attempt success cannot be achieved in approximately $50 \%$ of patients for PIVC. ${ }^{12}$ In the presence of DVA, patients are exposed to multiple interventions, resulting in increased pain levels. ${ }^{8,14}$ Thus, being successful in the first attempt is important in patients undergoing PIVC. As an alternative to the standard method, it is desired to increase the success rates by using technological devices, such as ultrasound and NIR.

A current meta-analysis comparing standard technique and ultrasound indicates that PIVC success can be increased with ultrasound in patients after multiple failed attempts. It is also emphasized that ultrasound is superior to the standard approach in decreasing puncture number and achieving success in a shorter time and provides advantages for patients with difficult or moderate vascular access features rather than its routine use. ${ }^{15-17}$ In studies conducted in intensive care patients and patients with DVA features, $71 \%-81 \%$ success in the first trial has been reported with ultrasound. ${ }^{16,18}$ Although the use of ultrasound in patients with DVA is generally considered superior to the standard method, it is also reported that there is no difference in terms of success, duration, and patient satisfaction. ${ }^{19}$ In this study, similar to the literature data, the first attempt success was achieved in $78 \%$ of patients undergoing PIVC with ultrasound. In terms of first attempt success, ultrasound was superior to standard and NIR methods. However, despite having DVA features of patients, the higher first attempt success rate $(62 \%)$ of the standard technique based on literature data may be related to nurses' experience level. This may also have caused no difference among groups (ultrasound and standard) regarding the total number of attempts and the need for rescue methods. In the future, studies with nurses of different seniority levels could clarify these situations.

Several studies were conducted in which emergency nurses performed vascular access procedures using ultrasound. In these studies, it was stated that nurses trained in ultrasound could perform the procedure as successfully as emergency residents. ${ }^{20}$ When the ultrasound-guided procedure is performed in patients with DVA, the success rate has increased, and the total procedure time has decreased. ${ }^{21}$ At the same time, the frequency of needing physician 


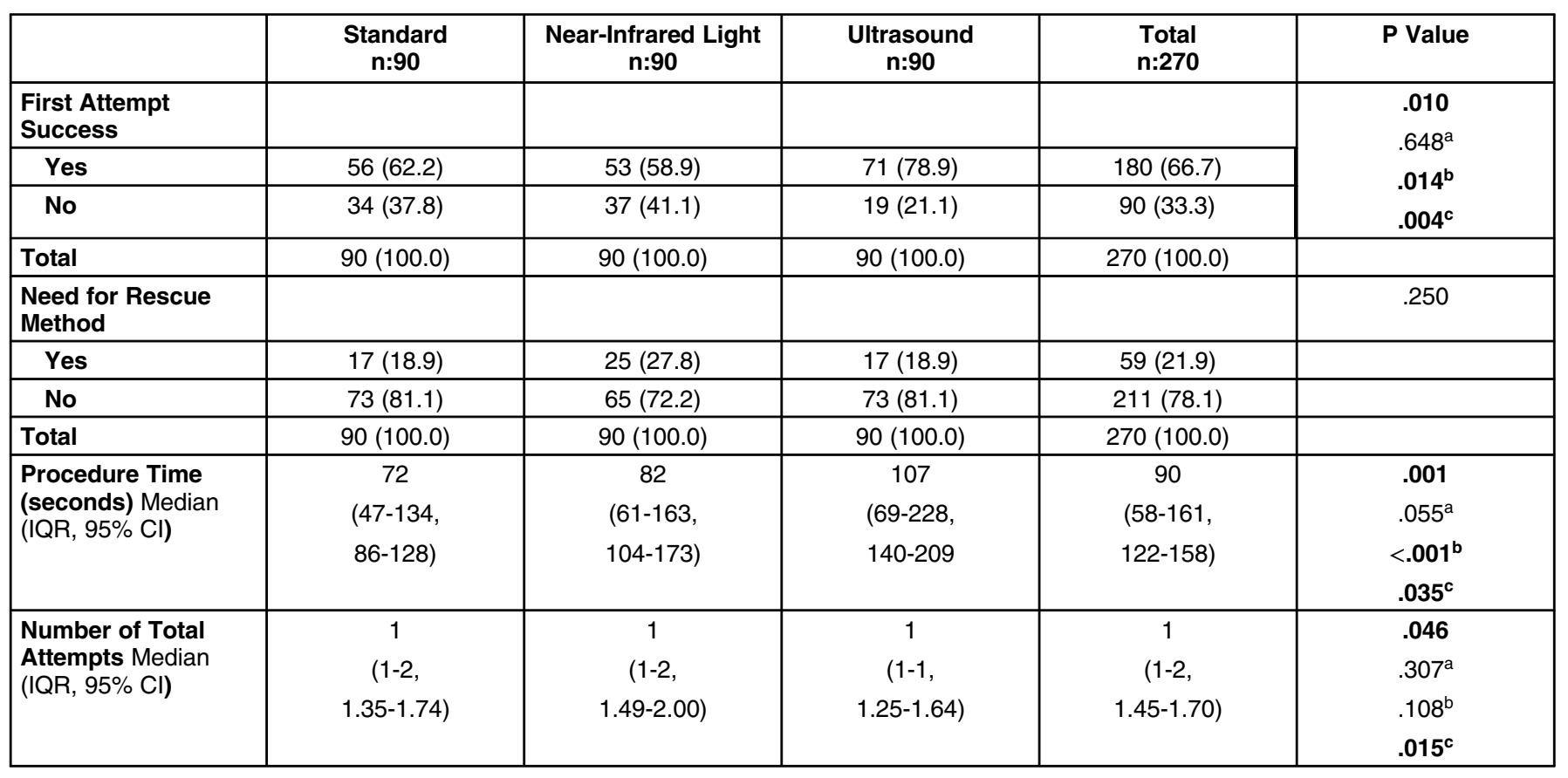

Table 2. Comparison of Study Outcomes Between Patient Groups

Yalçınlı (C 2022 Prehospital and Disaster Medicine

a Standard versus Near-Infrared Light.

${ }^{\mathrm{b}}$ Standard versus Ultrasound.

${ }^{\mathrm{c}}$ Near-Infrared Light versus Ultrasound.

intervention has decreased with the application of the procedure by nurses with the help of ultrasound in patients with DVA. ${ }^{9}$ Considering the literature data, it is understood that nurses who perform the PIVC procedure most frequently in clinical practice should be trained in obtaining vascular access with ultrasound to overcome the difficulties during DVA. ${ }^{22}$ There are various propositions about the content of this training. Moore, et al reported that nurses could reach $\geq 80 \%$ success by performing at least 25 successful procedures under supervision after four hours of didactic and practical training to become independent ultrasound practitioners. ${ }^{23}$ In the study of Feinsmith, et al, after four hours of theoretical and practical training, nurses who performed ten successful procedures under the supervision of a physician or nurse experienced in the use of ultrasound were evaluated. The first attempt and overall success rates were determined as $68 \%$ to $81 \%$ after 1-10 attempts and $87 \%$ to $96 \%$ after $21-30$ attempts, respectively. ${ }^{9}$ In the current study, after four hours of theoretical and four hours of practical training, the senior nurses who performed ten successful procedures under supervision achieved a $78 \%$ and $84 \%$ first attempt and overall success rates with ultrasound, respectively. The fact that ultrasound was the most preferred rescue method in this study suggests that nurses adopted the ultrasound-guided method. However, unlike the literature, the procedure time was longer than other methods, suggesting that nurses did not gain sufficient dexterity experience. This suggests that the number of ten exams determined in this study should be increased for nurses who did not have sufficient experience in the use of ultrasound before.

Because the infrared light device offers ease of use compared to ultrasound and can be applied after relatively brief training, it seems attractive for the health care professionals who need assistant devices in the presence of DVA. However, there are no studies on the use of NIR in the presence of DVA in adult patients. A study involving non-selected emergency department adult patients compared to the standard method reported that using an infrared light device did not differ in terms of the number of attempts, first attempt success, operation success, time to successful catheter placement, and procedure pain. In addition, in the same study, it is also emphasized that the infrared light device should be investigated in adult patients with DVA. ${ }^{24}$ Almost all of the data related to NIR are related to studies in the pediatric age group. In these studies, there are conflicting data about the routine use of the NIR device. A positive effect on first attempt success is not reported on overall pediatric patients, but it is reported that it may be beneficial in patients with DVA. But no evidence clearly recommends the use of NIR routinely. ${ }^{10,25-28}$ The current study did not determine the superiority of NIR over ultrasound or standard method in adult patients with DVA. Therefore, this study does not recommend using NIR to achieve success in the first attempt in adult patients with DVA. This needs to be investigated in future studies.

\section{Limitations}

This study conducted in a single-center is a limitation. Considering that other nurses would not have time to study due to workload, only senior nurses were included in the study. This situation may have limited the evaluation of the results from the variation in seniority among nurses. As a method of rescue, preferring ultrasound more frequently among nurses may have created a bias due to the nature of the study. The fact that the device getting time was not included in patients who underwent the procedure using USG 
and NIR may have resulted in a shorter procedure time in these methods. Compared with other studies in the literature, this study had a sufficient sample size, which increases the strength of the research.

\section{Conclusion}

Ultrasonography increases the success of the first attempt compared with the standard method and NIR in patients with DVA. Even nurses who have no previous experience with ultrasound can perform this procedure successfully after a short training and increase their skill. It was found that NIR does not constitute an advantage in procedure success in adult patients with DVA. These results do not support the use of NIR for adult PIVC.

\section{Acknowledgments}

The authors are grateful to Ege University Planning and Monitoring Coordination of Organizational Development and Directorate of Library and Documentation for their support in editing and proofreading service of this study.

\section{Supplementary Materials}

To view supplementary material for this article, please visit https:// doi.org/10.1017/S1049023X21001217

\section{References}

1. Fields MJ, Piela NE, Au AK, Ku BS. Risk factors associated with difficult venous access in adult ED patients. Am J Emerg Med. 2014;32(10):1179-1182.

2. Keyes LE, Frazee BW, Snoey ER, Simon BC, Christy D. Ultrasound-guided brachial and basilic vein cannulation in emergency department patients with difficult intravenous access. Ann Emerg Med. 1999;34(6):711-714.

3. Bauman M, Braude D, Crandall C. Ultrasound-guidance vs. standard technique in difficult vascular access patients by ED technicians. Am J Emerg Med. 2009;27(2):135-140.

4. Panebianco NL, Fredette JM, Szyld D, Sagalyn EB, Pines JM, Dean AJ. What you see (sonographically) is what you get: vein and patient characteristics associated with successful ultrasound-guided peripheral intravenous placement in patients with difficult access. Acad Emerg Med. 2009;16(12):1298-1303.

5. Au AK, Rotte MJ, Grzybowski RJ, Ku BS, Fields MJ. Decrease in central venous catheter placement due to use of ultrasound guidance for peripheral intravenous catheters. Am J Emerg Med. 2012;30(9):1950-1954.

6. Yalçınlı S, Akarca Karbek F, Can Ö, Şener A, Akbinar C. Factors affecting the firstattempt success rate of intravenous cannulation in older people. J Clin Nurs. 2019;28(11-12):2206-2213.

7. van Loon FHJ, Puijn LAPM, Saskia H, Bouwman ARA. Development of the A-DIVA scale: a clinical predictive scale to identify difficult intravenous access in adult patients based on clinical observations. Medicine (Baltimore). 2016; 95(16):e3428.

8. Witting MD. IV access difficulty: incidence and delays in an urban emergency department. J Emerg Med. 2012;42(4):483-487.

9. Weiner SG, Sarff AR, Esener DE, et al. Single-operator ultrasound-guided intravenous line placement by emergency nurses reduces the need for physician intervention in patients with difficult-to-establish intravenous access. $J$ Emerg Med. 2013;44(3):653-660.

10. Parker SIA, Benzies KM, Hayden KA, Lang ES. Effectiveness of interventions for adult peripheral intravenous catheterization: a systematic review and meta-analysis of randomized controlled trials. Int Emerg Nurs. 2017;31:15-21.

11. Von Elm E, Altman DG, Egger M, Pocock SJ, Gøtzsche PC, Vandenbroucke JP; STROBE Initiative. The Strengthening the Reporting of Observational Studies in Epidemiology (STROBE) statement: guidelines for reporting observational studies. J Clin Epidemiol. 2008;61(4):344-349.

12. Idemoto BK, Rowbottom JR, Reynolds JD, Hickman RL. The AccuCath intravenous catheter system with retractable coiled tip guidewire and conventional peripheral intravenous catheters: a prospective, randomized, controlled comparison. J Assoc Vasc Access. 2014;19:94-102.

13. Feinsmith S, Huebinger R, Pitts M, Baran E, Haas S. Outcomes of a simplified ultrasound-guided intravenous training course for emergency nurses. J Emerg Nurs. 2018;44(2):169-175.e2.

14. Fields MJ, Piela NE, Ku BS. Association between multiple IV attempts and perceived pain levels in the emergency department. J Vasc Access. 2014;15(6):514-518.

15. Liu YT, Alsaawi A, Bjornsson HM. Ultrasound-guided peripheral venous access: a systematic review of randomized-controlled trials. Eur J Emerg Med. 2014;21(1):18-23.

16. McCarthy ML, Shokoohi H, Boniface KS, et al. Ultrasonography versus landmark for peripheral intravenous cannulation: a randomized controlled trial. Ann Emerg Med. 2016;68(1):10-18.

17. van Loon FHJ, Buise MP, Claassen JJF, Dierick-van Daele ATM, Bouwman ARA. Comparison of ultrasound guidance with palpation and direct visualization for peripheral vein cannulation in adult patients: a systematic review and meta-analysis. $\mathrm{Br} \mathrm{J}$ Anaesth. 2018;121(2):358-366.

18. Gregg SC, Murthi SB, Sisley AC, Stein DM, Scalea TM. Ultrasound-guided peripheral intravenous access in the intensive care unit. J Crit Care. 2010;25(3):514-519.

19. Stein J, George B, River G, Hebig A, McDermott D. Ultrasonographically guided peripheral intravenous cannulation in emergency department patients with difficult intravenous access: a randomized trial. Ann Emerg Med. 2009;54(1):33-40.

20. Carter T, Conrad C, Wilson JL, Dogbey G. Ultrasound guided intravenous access by nursing versus resident staff in a community-based teaching hospital: a "noninferiority" trial. Emerg Med Int. 2015;2015:563139.

21. Bahl A, Pandurangadu AV, Tucker J, Bagan M. A randomized controlled trial assessing the use of ultrasound for nurse-performed IV placement in difficult access ED patients. Am J Emerg Med. 2016;34(10):1950-1954.

22. Smith C. Should nurses be trained to use ultrasound for intravenous access to patients with difficult veins? Emerg Nurse. 2018;26(2):18-24.

23. Moore C. An emergency department nurse-driven ultrasound-guided peripheral intravenous line program. J Assoc Vasc Access. 2013;18:45-51.

24. Aulagnier J, Hoc C, Mathieu E, Dreyfus JF, Fischler M, Le Guen M. Efficacy of AccuVein to facilitate peripheral intravenous placement in adults presenting to an emergency department: a randomized clinical trial. Acad Emerg Med. 2014;21(8):858-863.

25. Kuo CC, I-Jung F, Wei-Jing L. The efficacy of near-infrared devices in facilitating peripheral intravenous access in children: a systematic review and subgroup metaanalysis. Hu li za zhi. 2017;64:69-80.

26. Park JM, Kim JM, Hyeon WY, Lee W-C, Jeong H, Na JK. Utility of near-NIR devices for pediatric peripheral intravenous cannulation: a systematic review and metaanalysis. Eur J Pediatr. 2016;175(12):1975-1988.

27. Kim MJ, Park JM, Rhee N, et al. Efficacy of VeinViewer in pediatric peripheral intravenous access: a randomized controlled trial. Eur J Pediatr. 2012;171(7):1121-1125.

28. Heinrichs J, Fritze Z, Klassen T, Curtis S. A systematic review and meta-analysis of new interventions for PIVC intravenous cannulation of children. Pediatr Emerg Care. 2013;29(7):858-866. 